\title{
On the road to refinement
}

\author{
'Good welfare is good science,' the saying goes. But how do researchers, veterinarians, and animal care staff refine \\ the lives of their laboratory animals?
}

\section{Ellen P. Neff}

n 1997, Trevor Poole wrote that "Happy animals make good science," in response to what was, even more than two decades ago, a growing body of literature linking laboratory animal welfare to scientific outcomes. 'Happiness' is a state of mind, but Poole considered happy animals as those that "are busy, confident, and behav[ing] normally. They will not be in pain, or distressed, and should resist disease and reproduce successfully."

Striving for good animal welfare is important for the animal's sake. "It's the right thing to do," says Tara Martin, director of the Refinement \& Enrichment Advancements Laboratory (REAL) at the University of Michigan. "The animals didn't ask to be here...so we owe it to them to give them the best lives we possibly can."

There are also scientific implications if your animal is unwell due to its environment. "We need good welfare because we need good science," says University of Surrey veterinarian \& researcher Sarah Wolfensohn. An animal modeling a specific disease has that disease (or some semblance thereof), she notes; to study disease progression or determine if an intervention is having any beneficial effect, you don't want a welfare-related issue complicating your study. These issues can include stereotypies, subclinical disease, and abnormal behavior that are a consequence of the animals' environment - not the experiment itself.

Today, 'good welfare equals good science' is a common refrain that motivates efforts to refine research with laboratory animals that is, to reduce pain and distress while also introducing positive elements to their lives. But when your subjects can't speak, how do you know that what you are doing in the name of their welfare is working?

\section{The problem of pain}

A 'traditional' definition of welfare is all about the absence of negatives: pain, stress, suffering. As guidelines were released, accreditation programs developed, and animal welfare laws came on to the books in countries around the world in recent decades, the consideration and mitigation of negative impacts became increasingly

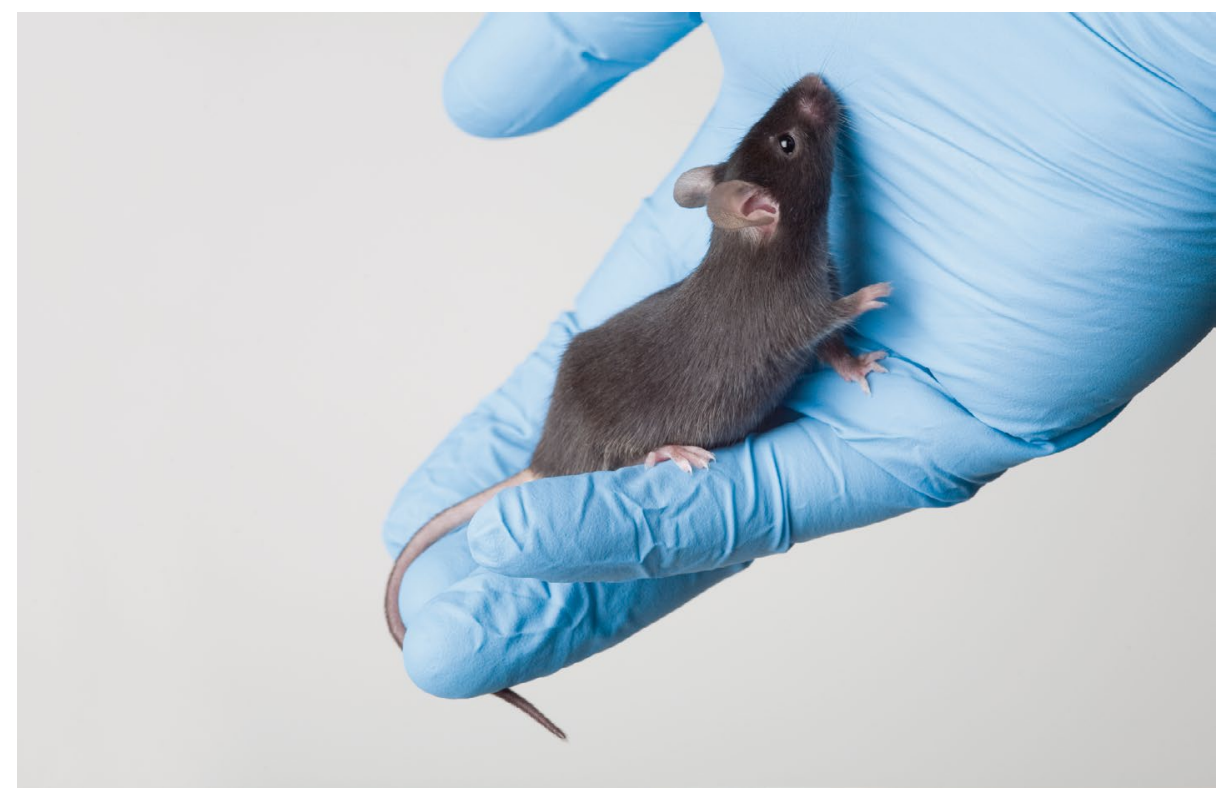

Handle with care | Concern for laboratory animal welfare - both minimizing negatives and increasing positives - continues to grow. Credit: dra_schwartz / E+ / Getty

important to veterinarians, researchers, and care staff working with laboratory animals. Providing analgesics - once rare - became the norm, while additional attention has been paid to pre-, peri-, and post-operative care for animals that require surgeries.

Much work has been (and continues to be) done to identify appropriate analgesics and anesthetics for different animals, but pain does present a pesky problem: how do you know that an animal is in pain when it can't tell you?

Endpoints are a proxy, but these can be a bit blunt - for example, severe weight loss, minimal movement, or coat changes. "All those are measures of welfare, but they tend to be fairly extreme and not very sensitive," says Daniel Pang, a veterinary anesthesiologist at the University of Calgary who studies anesthesia, analgesia, and pain in different species. "By the time you spot the problem, the animal has probably gone through a period of pain or stress." With more refined evaluations, you can identify pain - and act to mitigate it - earlier. That's good for the welfare of the animal, Pang says, and for experiments; for example, earlier identification of pain during a cancer study can reveal more granular information about what the animal is experiencing.

One promising means to identify pain that's been spreading through the literature over the past decade is the concept of a grimace scale, originally developed for use in mouse ${ }^{1}$ and rats $^{2}$ by Jeffrey Mogil's lab at McGill University. The idea is that by looking at the facial features of an animal whether it is squinting its eyes or changing the positioning of its whiskers, for example - you can determine how much pain it is experiencing.

That idea that that could work was a major step in welfare that required new openness towards the animals, says Pang. "It wasn't that long ago that the basic idea that looking at an animal's facial features seemed totally bizarre - why would you be able to expect to see a meaningful change in the face of a mouse or a rat?" he says.

Since the original rodent scales were published, grimace scales have been developed and validated for additional 
animals including pigs, rabbits, ferrets, guinea pigs, cats, horses, sheep, and cattle $\left(\right.$ see Review ${ }^{3}$ ), while for macaques, researchers have used Facial Action Coding Systems (FACS) ${ }^{4}$, a similar concept to grimace scales borrowed from human research, and other combinations of behavioral \& facial analyses (for example, see ref. ${ }^{5}$ ).

Grimace scales are now valuable research tools, but there are some practical issues that have limited their clinical applications ${ }^{3}$. Scoring pain with a scale does take a time, for both training the observer and to then analyze the faces of all those animal, which is often done from video recordings. In a research setting, you can dedicate the hours to that kind of data crunching, says Pang, but time and resources are tighter when you have to make animal care decisions at scale.

Video analysis has the potential for automation, and different groups have applied various machine learning algorithms to reduce the manpower needed ${ }^{6,7}$. While a step forward, those are still retrospective analyses; machine learning approaches continue to be refined, and in the meantime there are other efforts to bring the scales cage-side. Pang's lab is one group working on real-time applications; by comparing the performance of cage-side scores to retrospective scores from concurrently recorded videos, they and collaborators have shown that the scales have cage-side potential for both rats ${ }^{8}$ and cats ${ }^{9}$ so far.

Other methods for assessing pain take advantage of an animals' natural behavior. For example, mice in pain won't make the same use of nesting material as those without. Grooming might also be disrupted - at the REAL lab, they have experimented with adding a small dot of inert, fluorescent gel to the backs of their rodents; a quick flash of a black light can reveal if the mice have been grooming normally and spread that gel around. A mouse that isn't nesting or grooming properly may need a closer look ${ }^{10}$, says REAL Project Lead Sarah Thurston.

At the end of day, whatever approach is chosen needs to be realistic, and you need to make the case that others can succeed when adopting something new. "You can have the best scale in the world but if it takes more than a couple minutes...people just won't use it - it has to be practical," Pang says.

\section{Pursuing positives}

The animal research community has come a long way in reducing and managing negative effects on the animals, but animal welfare isn't just about avoiding negativity. "We now try to have that more holistic viewpoint and try to promote the positive state as well," says Alexandra Whittaker, a veterinarian, researcher, and animal research legal expert at the University of Adelaide in Australia. As with negating negatives, promoting positives comes with challenges too.

"In reality, the practical way of doing it is through an understanding of animal behavior," says Whittaker. That means knowing what normal behavior is, and how well the lab environment can provide animals the option to behave that way - are social animals housed together? Are there shelters to hide in, enrichment devices to play with, or nesting materials to burrow into?

That all depends on the animal you are considering. What's 'normal' for mice will differ from macaques will differ from zebrafish - you will need to know your animal, and there is no 'one size fits all. "We try to leverage the species-specific, natural behaviors of our animals to learn more about them and let them 'tell' us how they are feeling," says Thurston. Any time they take on a project to evaluate whether a potential refinement is indeed beneficial to a given animal at Michigan, they

start by putting together a review of its natural history.

Regular welfare monitoring from technicians, vets, and researchers is key and includes assessments of variables such as the animal's appearance, body functions, and behavior, as well as its environment and any procedure-related indicators. As these assessments can bear some level of subjectivity, there have been attempts to provide objective measures that capture the state of animal's welfare, and to then track it over time.

In 2007, Wolfensohn came up with the concept of the animal welfare assessment grid (AWAG), which is based on four main parameters: physical well-being, behavioral/ physiological well-being, environmental conditions, and experimental \& clinical events. Each parameter is divided into species-relevant subfactors, which can receive a score from 1 (best) to 10 (worst). The combined scores produce a graphical representation of the animals' welfare.

The AWAG was adopted by Public Health England to assess the welfare of the nonhuman primates it uses for vaccine studies $^{11}$; the group also produced a software package available on Github as a step up from calculating the scores via Excel. Recently, Wolfensohn teamed up with a software company to develop a graphical user interface for the AWAG in the hopes of facilitating greater adoption. "It has to be quick to use - you just click the button," she says. The current software contains grids for dogs, horses, zoo animals, and farm animals; one of Wolfensohn's students will be working on the criteria to add lab animals, including rats, mice, zebrafish, and Xenopus.

The idea is to track the animals' welfare over time. Day to day, things might not change all that much, but there is a growing body of evidence that cumulative experiences can add up.

Judgement bias tasks have emerged as a means to measure an animals' affective valence - in animal terms, whether it is in a positive or negative state (or to anthropomorphize, whether it is feeling more optimistic or pessimistic about its life). The idea is to test the animals' decision making in the face of ambiguity. For example, an animal may be taught to associate a particular tone or visual stimulus with a positive outcome, such as getting a treat, and another with a negative outcome, such as aversive puff of air. When the stimulus is ambiguous, whether the animal reacts in a positive or negative way can reflect is affective valence. Cognitive bias has been demonstrated in animals including
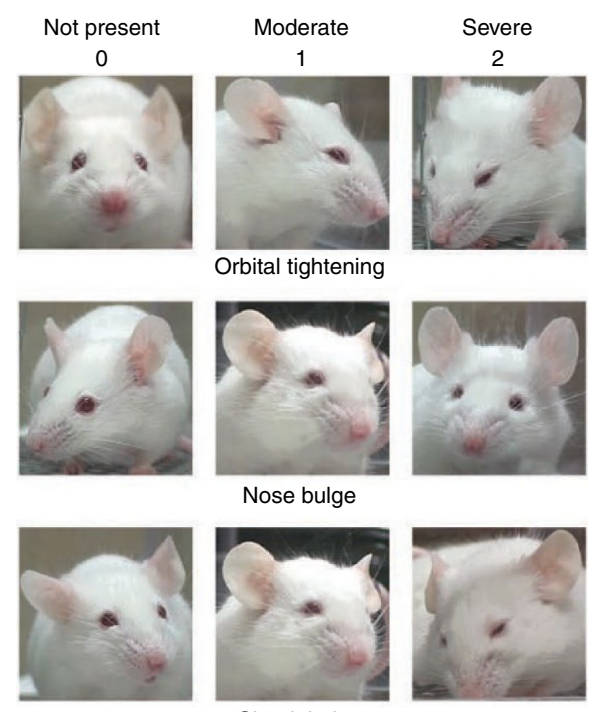

Cheek bulge
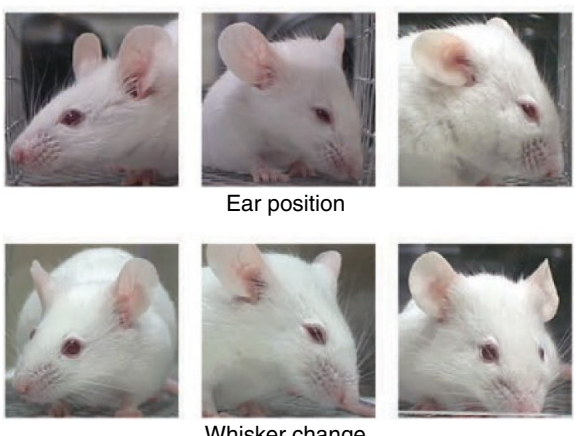

A tell-tale countenance | The grimace scales evaluate facial features of different animals, such as these mice, to assign a pain score. Image reprinted with permission from Langford (Springer Nature) $2010^{1}$. 


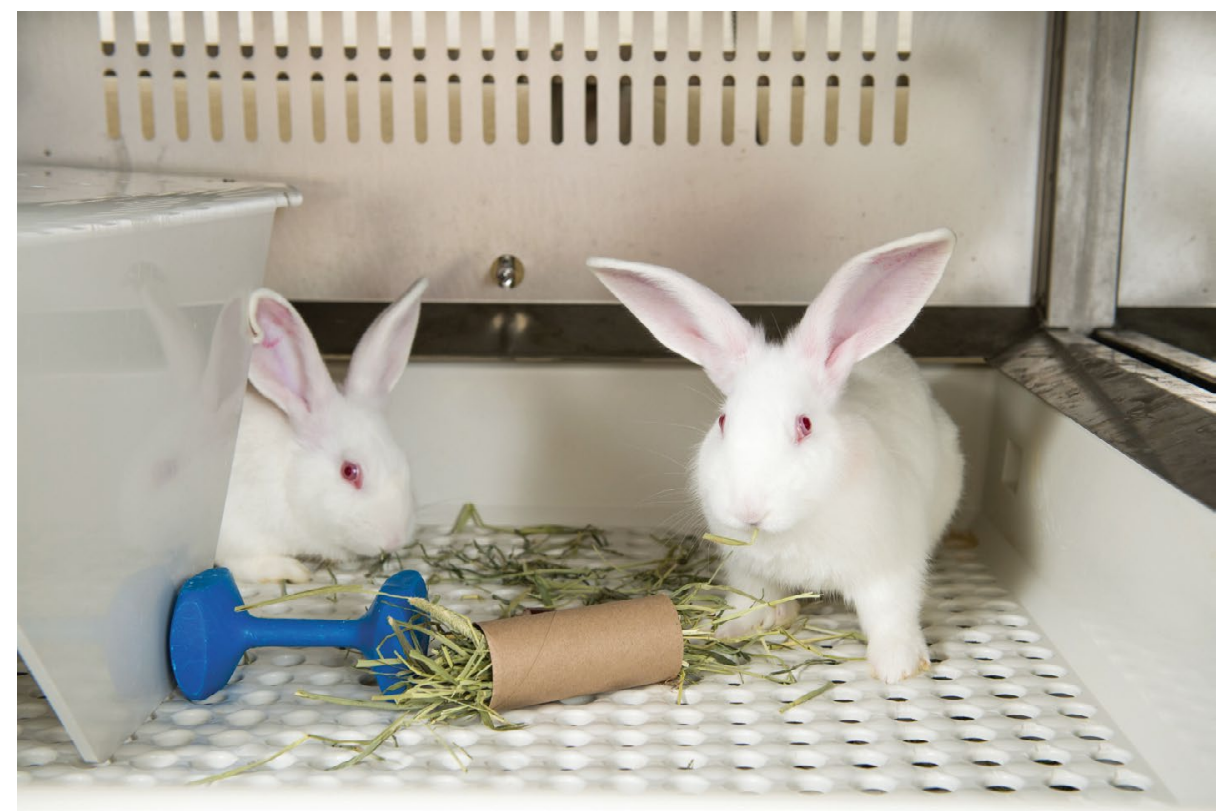

Not a crowd | Rabbit pairing made possible with refinement. Credit: Austin Thomason, Michigan Photography - University of Michigan

rodents, canines, macaques, and marmosets in recent years.

Although such bias tasks have become valuable research tools, work still needs to be done to move them to the animal facility. "The challenge is how you do that on a practical, day to day basis, because it takes a long time to train animals," says Whittaker. As with the grimace scales, computers will likely help, and there have been some studies evaluating automated judgement bias tests ${ }^{12}$. Custom home cages and home cage-based devices, such as touchscreens ${ }^{13}$, may also help researchers refine an animals' experience.

"I think we'll get there, but we need to get to the stage where some of these tests are being automated, to take out the human element and when you just don't have time in your day," says Whittaker.

\section{Building best practices}

Assessing an animal's welfare is important and an area that has indeed improved in the laboratory animal space, but the task doesn't stop there. "Just having it written down and checking the boxes isn't enough," says Wolfensohn. "You have to review what you're doing and make changes."

That means figuring out what actually is best for the animal. There, simple but scientifically valid experiments can be set up to compare how an animal responds to a different say, analgesic, form of enrichment, handling method, or housing set up. For example, does the change expand their repertoire of species-normal behavior, or reduce physiological measures of stress, such as cortisol, says Whittaker.

While there are increasingly sophisticated tools to record and monitor animals automatically, and often in the comfort of questions, the day-to-day still needs those practical best practices, says Jennifer Logren, a researcher now at Novartis who helped found the REAL Lab in 2014.

Take mouse handlings, traditionally done via the tail. Work from Jane Hurst's lab and others has shown that this is however a bit stressful for the animals; using cupped hands or a small plastic tunnel is a simple improvement. To administer drugs, staff can consider ingestible approaches, rather than injections ${ }^{14}$. Adding nesting material that allows them to burrow can alleviate stress, and help mice thermoregulate - which have consequences for metabolic-related research as well as 'cold' mice will respond different than warmer animals ${ }^{15}$. Some rats just like to be tickled. "Areas where we are starting to make progress and need to continue is the consideration of the lived experience of the animal on study. Their experience is influenced by so many things that can seem incidental to people, but can have a huge impact on animal welfare," says Lofgren.

At REAL, particular project often come from a clinical problem, or from an idea born from staff working with the animals, says Martin. The lab is dedicated to producing evidence to support animal care refinements, and its staff are cognizant of experimental design considerations and the home cage, that are valuable for research statistical significance - the kind of evidence important to the researchers working with the animals. "Science is a universal language. Scientists want to do the right thing by the animals and they're trying to bring new therapies and hope to patients," says Lofgren. "Having a mechanism, be it a lab or a program, to support 3Rs advancements allows us to partner with scientists so they can have the data they need to confidently adopt new practices."

Simple solutions go a long way. Rabbits, for example, have a tendency to urine spray; it's normal behavior, but not the most hygienic, says Martin. With a little extra monitoring, the REAL staff determined that they could prevent excess spraying by switching the animals' neighbors. "It's a small, simple change, but it's easy to implement," she says. With some husbandry tweaks, they've also had considerable success in pair-housing their rabbits - feat thought to be impossible by many ${ }^{16}$.

Time \& resources are limited, so easy solutions can ease buy in, which all adds up for the animals. "All of the research you do in refinement and enrichment - it builds," says Martin. The literature continues to grow.

Over the last 30 years, animal welfare has come a long way, says Wolfensohn. The 3Rs has taken off, with dedicated groups around the world promoting and, as they can, funding efforts to replace, reduce, and refine animal use. The last $\mathrm{R}$ remains critical. "The reality is, animals are going to continue being used in science, because we do need animal models," she says. "Whatever we do, do it better."

\section{Ellen P. Neff ${ }^{凶}$ \\ Lab Animal. \\ $\bigotimes_{e-\text { mail:ellen.neff@us.nature.com }}$}

Published online: 10 September 2021 https://doi.org/10.1038/s41684-021-00854-4

\footnotetext{
References

1. Langford, D. J. et al. Nat Method 7, 447-449 (2010).

2. Sotocinal, S. G. et al. Mol. Pain 7, 55 (2011).

3. Mota-Rojas, D. et al. Animals 10, 1838 (2020).

4. Parr, L. A., Waller, B. M., Burrows, A. M., Gothard, K. M. \& Vick, S. J. Am J. Phys Anthropol 143, 625-630 (2010).

5. Descovich, K. A. et al. Altex 36, 535-554 (2019).

6. Tuttle, A. H. et al. Mol. Pain 14, 174480691876365 (2018).

7. Andresen, N. et al. PLoS One 15, e0228059 (2020).

8. Leung, V., Zhang, E. \& Pang, D. S. J. Sci. Rep. 6, 31677 (2016).

9. Evangelista, M. et al. PeerJ https://doi.org/10.7717/peerj.8967 (2020).

10. Oliver, V. L., Thurston, S. E. \& Lofgren, J. L. J. Am Assoc. Lab. Anim. Sci. 57, 180-201 (2018).

11. Wolfensohn, S. et al. Animal Welfare 24, 139-149 (2015).

12. Jones, S. et al. Sci Rep. 8, 12400 (2018).

13. Lopez-Crus, L., Bussey, T. J., Saksida, L. M. \& Heath, C. J. Lab. Anim. (NY) 50, 174-184 (2021).

14. Schalbetter, S. M. et al. Lab. Anim. (NY) 50, 69-75 (2021) 15. Landhuis, E. Lab. Anim. (NY) 47, 119-121 (2018)

16. Thurston, S., Burlingame, L., Lester, P.A., and Lofgren, J. JOVE https://doi.org/10.3791/57267 (2018).
} 